segmented AAPC for YIR was $-0.63 \%(p=0$. 0.0048) before 2014 and $-3.71 \%(p=0.0 .0596)$ after 2014. Segmented YRR AAPC was $-0.07 \% \quad(p=0.67)$ before 2011 and $+1.24 \%$ $(\mathrm{p}=0.0006)$ after 2011. An 8-year latency between YIR decrease and significant YRR change was observed. In 2017, the YRR (20.6\%) surpassed the YIR (19.7\%) for the first time. YIRs had a strong inverse correlation with YRRs $(\mathrm{PC}=-$ $0.82, \mathrm{p}=0.0002$; figure $1 \mathrm{~B}$ ).

Conclusion Following ARUBA, there has been a stark decrease in interventions for uAVMs. Nationwide rAVM discharge incidence is inversely correlated with uAVM intervention rate. Less uAVM interventions may lead to a substantial increase in AVM rupture incidence.

Disclosures D. McCarthy: None. E. Luther: None. B. Gross: None. R. Starke: None.

\section{LB-006 INCREASED INTRACRANIAL AND SYSTEMIC VCAM1 RELATES TO HYPERTENSION AND REDUCED PERCENT CHANGE IN NIHSS AFTER MECHANICAL THROMBECTOMY}

'B Maglinger*, , $\mathrm{M}$ Sands, ${ }^{1} \mathrm{~J}$ Frank, ${ }^{1} \mathrm{~A}$ Trout, ${ }^{2} \mathrm{~J}$ Roberts, ${ }^{2} \mathrm{~S}$ Grupke, ${ }^{1} \mathrm{~J}$ Turchan-Cholewo, ${ }^{1} \mathrm{~A}$ Stowe, ${ }^{2} \mathrm{~J}$ Fraser, ${ }^{1} \mathrm{~K}$ Pennypacker. 'Department of Neurology, University of Kentucky, Lexington, KY; ${ }^{2}$ Department of Neurosurgery, University of Kentucky, Lexington, $K Y$

\subsection{6/neurintsurg-2020-SNIS.278}

Introduction The University of Kentucky Blood and Clot Thrombectomy Registry and Collaboration (BACTRAC) protocol (clinicaltrials.gov NCT03153683) utilizes thrombectomy to isolate intracranial (i.e. distal to thrombus) arterial blood and systemic (i.e. carotid) arterial blood from thrombectomy procedures to better understand stroke. The objective of this study within that protocol was to investigate the relationship among Vascular Cell Adhesion Molecule 1 (VCAM1), hypertension (HTN), and stroke recovery in patients undergoing mechanical thrombectomy for emergent large vessel occlusion (ELVO) stroke. Here, we report how intracranial and systemic proteomic expression levels relate to HTN and the change in National Institute of Health Stroke Scale/Score (NIHSS) from admittance to discharge.

Methods Intracranial and systemic plasma samples from 25 subjects underwent cardiometabolic proteomic analysis at Olink Proteomics (Olink Proteomics, Boston, MA). VCAM1 expression levels were reported as a Normalized Proteomic eXpressoin (NPX) for each subject. Demographic data were also reported including HTN and both admission NIHSS and discharge NIHSS. Linear regression analysis was run on both intracranial and systemic VCAM1 expression levels against $\%$ change in NIHSS ((Admittance NIHSS - Discharge NIHSS)/ Admittance NIHSS)). Two-tailed t-tests were run assessing VCAM1 expression with hypertension (HTN) vs. no hypertension. Data analysis was performed using IBM SPSS Statistics.

Results Linear regression analysis demonstrated increased expression of intracranial VCAM1 significantly correlated with a smaller\% change in NIHSS $(p=0.001)$. Similarly, increased systemic VCAM1 expression was also found to have a significant relationship with smaller\% change in NIHSS $(p=0.005)$. Subjects with hypertension had significantly higher intracranial $(p=0.03)$ and systemic $(p=0.001)$ VCAM1 levels compared to those without HTN.
Discussion VCAM1 mediates leukocyte-endothelial cell adhesion and has been shown to play a role in atherosclerotic plaques, endothelial integrity and function, and stroke recurrence. This study takes a novel approach of sampling both intracranial and systemic arterial blood during an ELVO stroke. We found increased intracranial and systemic VCAM1 independently correlate with a smaller\% change in NIHSS. Although preliminary, these results may suggest an informative role of VCAM1 levels at the time of infarct. Additionally, within our cohort, those with HTN had higher levels of intracranial and systemic VCAM1. These data are in line with previous studies suggesting VCAM1 may be a marker of endothelial damage due to HTN. To better understand our findings, we plan to perform subset analyses investigating VCAM1 levels in relation to stroke recurrence, dyslipidemia, infarct time and infarct volume. As VCAM1 plays a role in leukocyte recruitment during the inflammatory state, we also plan to investigate how the antioxidant superoxide dismutase 1 (SOD1) may modulate intracranial and systemic levels of VCAM1 and influence functional recovery based on NIHSS.

Disclosures B. Maglinger: None. M. Sands: None. J. Frank: None. A. Trout: None. J. Roberts: None. S. Grupke: None. J. Turchan-Cholewo: None. A. Stowe: 4; C; Cerelux, LLC. J. Fraser: 2; C; Stream Biomedical, Medtronic, Penumbra. 4; C; Cerelux, LLC; Fawkes Biotechnology. K. Pennypacker: 4; C; Cerelux, LLC.

\section{LB-007 RESULTS OF HYBRID STUDY, PROSPECTIVE RANDOMIZED MULTICENTER STUDY OF HYDROGEL COIL VS BARE PLATINUM COIL FOR INTRACRANIAL ANEURYSMS}

${ }^{1} \mathrm{~N}$ Sakai ${ }^{*},{ }^{1} \mathrm{H}$ Imamura, ${ }^{1} \mathrm{C}$ Sakai, ${ }^{2} \mathrm{~A}$ Hyodo, ${ }^{3} \mathrm{Y}$ Ito, ${ }^{4} \mathrm{Y}$ Matsumaru, ${ }^{5} \mathrm{~S}$ Miyachi ${ }^{6} \mathrm{~S}$ Yoshimura, ${ }^{7} \mathrm{M}$ Sasaki, ${ }^{8} \mathrm{~K}$ Ogasawara, ${ }^{9} \mathrm{~S}$ Miyamoto, ${ }^{9} \mathrm{~S}$ Miyamoto, ${ }^{10} \mathrm{M}$ Ezura, ${ }^{11}$ I Nakahara, ${ }^{9} \mathrm{~A}$ Ishii, ${ }^{12} \mathrm{~T}$ Higashi. ${ }^{1}$ Neurosurgery, Kobe City Medical Center General Hospital, Kobe, Japan; ${ }^{2}$ Neurosurgery, Dokkyo Medical Univesity Saitama Medical Center, Koshigaya, Japan; ${ }^{3}$ Neurosurgery, Shinrakuen Hospital, Niigata, Japan; ${ }^{4}$ Neurosurgery, Tsukuba University, Tsukuba, Japan; ${ }^{5}$ Neurosurgery, Aichi Medical University, Nagakute, Japan; ${ }^{6}$ Neurosurgery, Hyogo College of Medicine, Nishinomiya, Japan; ${ }^{7}$ Radiology, Iwate Medical University, Morioka, Japan; ${ }^{8}$ Neurosurgery, Iwate Medical Unuversity, Morioka, Japan; ${ }^{9}$ Neurosurgery, Kyoto University, Kyoto, Japan; ${ }^{10}$ Neurosurgery, Sendai Medical Center, Sendai, Japan; "Neurosurgery, Fujita Health University, Toyoake, Japan; ${ }^{12}$ Neurosurgery, Fukuoka University Chikushi Hospital, Chikushino, Japan

\subsection{6/neurintsurg-2020-SNIS.279}

Purpose To present results of HYBRID study, whether second generation hydrogel coils (HGC) for the treatment of intracranial aneurysms improves outcomes compared with bare platinum coils.

Material and Methods HYBRID study is investigator-initiated, prospective, randomized, multicenter trial with open label treatment, blinded endpoint evaluation in Japan (NCT01516658). Inclusion criteria are 20-80 age, 7-20 mm size, unruptured or ruptured with WFNS grade I-III. For hydrogel coil arm ( $\mathrm{H}$ group), recommended to use HGC $50 \%$ length or more with maximum effort and for bare platinum coil arm (B group) prohibited to use any HGC and surface modificated coil. Primary endpoint is recanalization, evaluated on DSA at 1-year after embolization, and late aneurysm rupture, re-treatment and adverse events are secondary endpoints. 
Results 431 cases enrolled in 44 centers in Japan since March 2012 to March 2016, and completed last follow-up on March 2017. Background data, age, gender, aneurysm size, dome/neck ratio, VER were not significant different in both arms, and mean and median length\% of used HGC was 68.6 and 69.9 in $\mathrm{H}$ Group and 1.2 and 0 in B Group. Primary endpoint in $\mathrm{H}$ group $(3.3 \%, 7 / 214)$ couldn't show significant difference $(\mathrm{p}=0.083)$ with that in $\mathrm{B}$ group (7.1\%, 15/211). However, evaluation by same definition with previously published report, showed major recanalization at 1 year after procedure in $\mathrm{H}$ group $(2.3 \%, 5 / 214)$ was significantly lower $(p=0.039)$ than B group $(6.6 \%, 14 /$ 211). And also, adverse event in $H$ group is significantly lower than that in B group and no aneurysm rupture in both groups.

Conclusion Second generation hydrogel coils decreases adverse outcomes including recanalization and unfavorable clinical events in endovascular treatment for intracranial aneurysms.

Disclosures N. Sakai: 1; C; Terumo/Microvntion, Medtronic, Neurovasc, Daiichi-Sankyo. 2; C; Asahi Intec, Biomedical Solutions, Johnson\&Johnson/Cerenovus, Medtronic, Neurovasc, Penumbra, Stryker, Terumo. H. Imamura: 2; C; Medtronic. C. Sakai: None. A. Hyodo: None. Y. Ito: None. Y. Matsumaru: None. S. Miyachi: None. S. Yoshimura: None. M. Sasaki: None. K. Ogasawara: None. S. Miyamoto: None. S. Miyamoto: None. M. Ezura: None. I. Nakahara: None. A. Ishii: None. T. Higashi: None.

\section{LB-008 MOTOR NEUROPROSTHESIS IMPLANTED USING CEREBRAL VENOGRAPHY IMPROVES ACTIVITIES OF DAILY LIVING IN SEVERE PARALYSIS}

${ }^{1} \mathrm{~T}$ Oxley, ${ }^{2} \mathrm{P}$ Mitchell ${ }^{*},{ }^{2} \mathrm{~N}$ Opie, ${ }^{2} \mathrm{P}$ Yoo, ${ }^{3} \mathrm{~J}$ Mocco, ${ }^{2} \mathrm{~B}$ Campbell, ${ }^{2} \mathrm{C}$ Bird, ${ }^{4} \mathrm{~S}$ Lee. ${ }^{1}$ University of Melbourne, Melbourne, Australia; ${ }^{2}$ Royal Melbourne Hospital, Melbourne, Australia; ${ }^{3}$ Mount Sinai Health System, New York City, NY; ${ }^{4}$ Calvary, Melbourne, Australia

\subsection{6/neurintsurg-2020-SNIS.280}

Background An implantable Brain Computer Interface (BCI) may function as a motor neuroprosthesis to restore voluntary motor function in patients with severe paralysis due to brain, spinal cord, peripheral nerve or muscle dysfunction, but to date demonstrations have been limited in clinical translation. We report the home-based use of a fully-implanted mobile motor neuroprosthesis using a novel endovascular stent-electrode, avoiding craniotomy, to restore voluntary motor control of a patient by means of a personal computer.

Methods The participant was a 75 -year-old man with flaccid paralysis in his upper limbs due to Motor Neuron Disease (MND). Prior to the study he was totally dependent on his caregiver wife for all Instrumental Activities of Daily Living (IADLs) including remote communication. Using routine cerebral venography techniques, the device was placed in the superior sagittal sinus adjacent to primary motor cortex and connected to a subcutaneous wireless transmitting unit. The participant underwent closed-loop training to convert
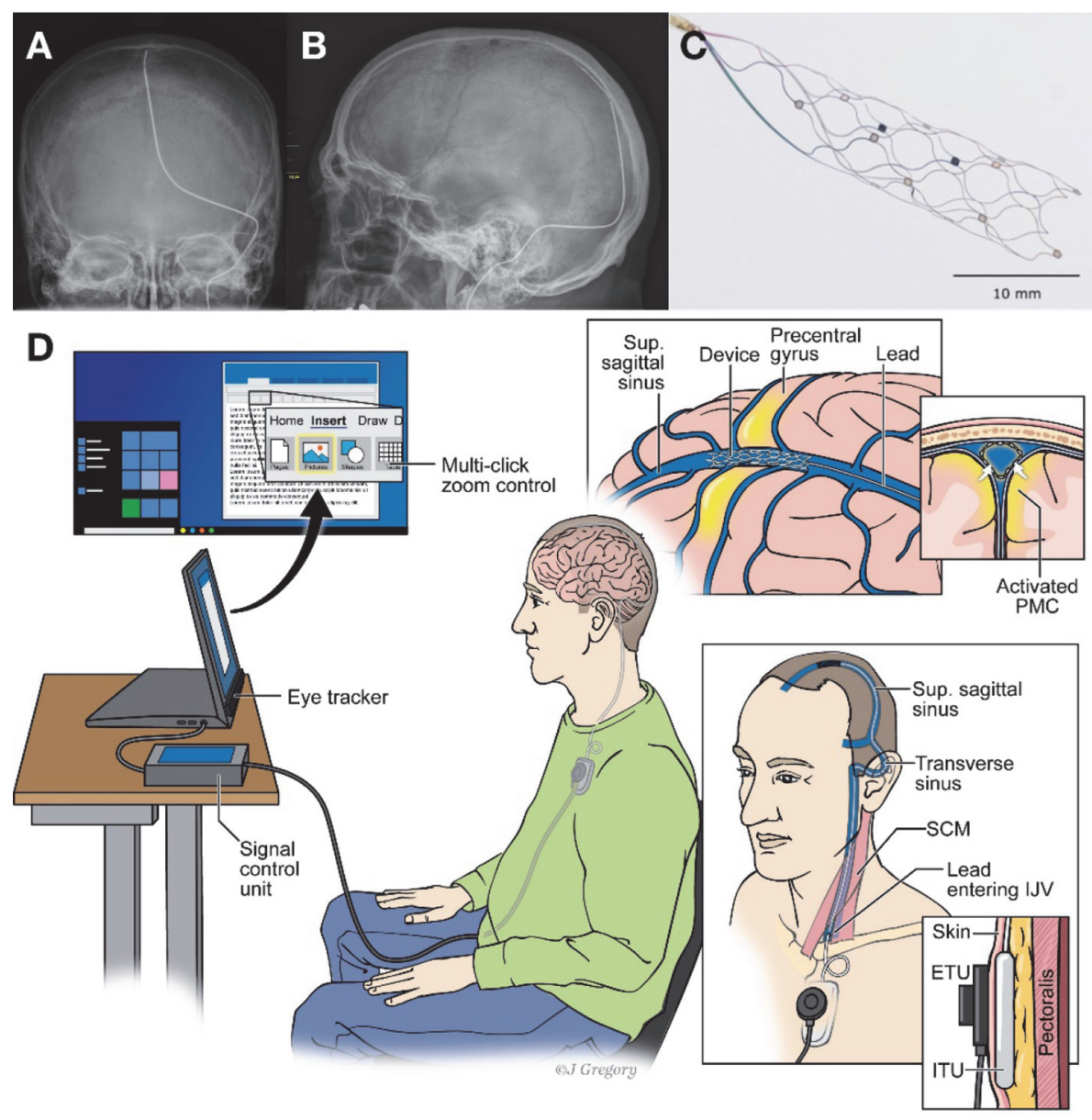

Abstract LB-008 Figure 1 Endovascular motor prosthesis system 